\title{
Activités antioxydantes des huiles essentielles de trois plantes aromatiques acclimatées au Togo
}

\author{
Kokou Agbékonyi AGBODAN*, Kokouvi DOTSE et Kossi Honoré KOUMAGLO \\ Laboratoire des Extraits Végétaux et Arômes Naturels (LEVAN), Faculté des Sciences, Université de Lomé, \\ BP 1515, Togo. \\ *Auteur correspondant ; E-mail : akatologo@yahoo.fr; Tél : (+228)90047531
}

\section{RESUME}

La présente étude s'inscrit dans le cadre de valorisation des HE des plantes aromatiques de notre sous région en tant que molécules bioactives possédant des propriétés antioxydantes. Dans ce contexte, l'objectif spécifique de ce travail est la recherche de la composition chimique des extraits de trois plantes acclimatées au Togo : Vetiveria zizanioides, Aeollanthus pubescens et Xylopia aethiopica et l'identification des échantillons qui ont des propriétés antioxydantes remarquables. LEVAN qui est le laboratoire des extraits végétaux et arômes naturels nous a servi de cadre. Deux méthodes sont appliquées pour mesurer le pouvoir antioxydant. Il s'agit du test de réduction de la 2,2-diphényl -1-picrilhydrazyle (DPPH) en utilisant la quercétine comme référence et du test de réduction de l'ion ferreux $\mathrm{Fe}^{2+}$ connu sous le nom de test « Ferric-Reducing Antioxidant Power »(FRAP). Les propriétés antioxydantes des HE ont été mesurées et mises en évidence par la recherche de l'intensité colorante $\left(\mathrm{IC}_{50}\right)$ qui représente la quantité de produit nécessaire pour faire diminuer la coloration de la solution de réactif (DPPH) de moitié, soit $50 \%$ et la détermination de l'équivalent en $\mathrm{Fe}^{2+}$ des solutions testées. Les constituants chimiques des HE sont identifiés par la chromatographie en phase gazeuse, par comparaison de leur temps de rétention, avec ceux des produits de référence publiés dans la littérature. L'extraction, par entraînement à la vapeur d'eau de matières végétales (racines et parties aériennes) de Vetiveria zizanioides (Poaceae) récolté à Zévé (Sud-Togo), d'Aeollanthus pubescens (Lamiaceae) récolté au centre du Togo, de Xylopia aethiopica (Annonaceae) acheté à Tsévié (Sud-Togo), donne de différents rendements en huiles essentielles. L'HE d'Ocimum basilicum est achetée en France. Les résultats obtenus, montrent que l'huile essentielle d'O. basilicum présente un pouvoir antioxydant supérieur, indépendamment de la méthode utilisée, soit une intensité colorante $\left(\mathrm{IC}_{50}\right)$ égale à $266 \mathrm{ppm}$ et l'équivalent en $\mathrm{Fe}^{2+}$ de 1318 $\mu$ mol. $\mathrm{L}^{-1}$. Il existerait une corrélation entre la capacité antioxydante des HE et leurs teneurs en composés phénoliques.

(C) 2014 International Formulae Group. All rights reserved.

Mots clés : Xylopia aethiopica, Aeollanthus pubescens, Vetiveria zizanioides, Ocimum basilicum à linalol, Huiles essentielles.

\section{INTRODUCTION}

Un antioxydant est « toute substance qui, présente à faible concentration comparée à celle du substrat oxydable, retarde ou prévient de manière significative l'oxydation de ce substrat ». On savait depuis que beaucoup d'antioxydants de synthèse généralement utilisés se sont avérés responsables d'effets indésirables et cancérigènes (Corinne, 2013 ; Hélène, 2008). 
L'innocuité des produits chimiques, utilisés en médecine et en industries agroalimentaire, cosmétique et pharmaceutique, constitue une grande préoccupation pour les chercheurs. Des études ont montré que certaines huiles essentielles ont des capacités antioxydantes remarquables contenant parfois une source naturelle de molécules bioactives (Djéridane et al., 2010; Benhammadou, 2011). La littérature mentionne beaucoup de méthodes conventionnelles d'étude du pouvoir antioxydant, telles que les tests Trolox Equivalent Antioxidant Capacity (TEAC), Ferric-Reducing Antioxidant Power (FRAP), Oxygen Radical Absorbance Capacity (ORAC) et le test à la 2,2-diphényl -1picrilhydrazyle (DPPH). Des analyses GC/MS et des mesures de la densité optique des solutions d'échantillons testés nous ont permis de déterminer la composition chimique et le potentiel antioxydant des huiles essentielles extraites de trois plantes aromatiques de la flore togolaise, et par comparaison d'une HE achetée en France. Nous avons utilisé les tests à la DPPH et au FRAP pour la détermination du pouvoir antioxydant.

\section{MATERIEL ET METHODES \\ Obtention du matériel}

Les racines de Vetiveria zizanioides et les feuilles d'Aeollanthus pubescens récoltées à l'intérieur du pays, les fruits secs de Xylopia aethiopica achetés sur le marché togolais en décembre 2009, sont lavés et séchés à l'ombre au laboratoire pendant 20 jours. Les échantillons d'huiles essentielles (H.E), à partir de ce matériel végétal, ont été extraits par entraînement à la vapeur d'eau (Kokouvi, 2009). La pureté de ces HE a été confirmée par la mesure de leurs indices de réfraction à l'aide d'un réfractomètre et leurs constituants chimiques sont identifiés par la chromatographie en phase gazeuse, par comparaison de leur temps de rétention, avec ceux des produits de référence publiés dans la littérature.
Préparation des réactifs et des huiles essentielles à tester : échantillonnage

Pour préparer le réactif du test FRAP, il faut mélanger trois solutions : le tampon acide $\mathrm{pH}=3,5(50 \mathrm{ml})$, la solution du 2,4,6tripyridyl-s-triazine (TPTZ) $(5 \mathrm{ml})$ et la solution de chlorure de fer III $(5 \mathrm{ml})$. Le protocole suivant est utilisé: pour le tampon acide ( $\mathrm{pH}=3,5)$, Il faut dissoudre $0,310 \mathrm{~g}$ de $\mathrm{CH}_{3} \mathrm{COONa}, 3 \mathrm{H}_{2} \mathrm{O}$ dans une fiole de $100 \mathrm{ml}$, ajouter ensuite 1,6 $\mathrm{ml}$ d'acide acétique pur et compléter avec de l'eau distillée jusqu'au trait de jauge. La Solution de TPTZ est préparée en dissolvant $156 \mathrm{mg}$ de ce composé dans $50 \mathrm{ml}$ de $\mathrm{HCl}$ à $40 \mathrm{mmol} / \mathrm{L}$. Pour la solution de chlorure de fer III $(20 \mathrm{mmol} / \mathrm{L})$, nous avons dissous $27 \mathrm{mg}$ de $\mathrm{FeCl}_{3}, 6 \mathrm{H}_{2} \mathrm{O}$ dans une fiole de $50 \mathrm{ml}$ et complété à l'eau distillée. La solution de sulfate de fer II pour le test FRAP, est préparée en dissolvant $27 \mathrm{mg}$ de $\mathrm{FeSO}_{4}, 7 \mathrm{H}_{2} \mathrm{O}$ dans $50 \mathrm{ml}$ de méthanol. La solution de concentration molaire $\mathrm{C}=2000$ $\mu$ mol..$^{-1}$ obtenue est diluée de manière à avoir plusieurs solutions de concentrations différentes de sulfate de fer II. Le réactif DPPH est une solution de la 2,2-diphényl -1picrilhydrazyle dans du méthanol, de concentration 100 micromoles par litre. Afin d'éviter la dégradation des produits avant leur utilisation, les (HE) ainsi que les réactifs DPPH et FRAP sont gardés au réfrigérateur à $4{ }^{\circ} \mathrm{C}$. Avant leur utilisation, ils sont sortis et laissés au laboratoire, à la température ambiante et à l'abri de la lumière. A $20 \mathrm{mg}$ de chaque échantillon d'HE, on ajoute du méthanol pur dans une fiole jaugée de $20 \mathrm{ml}$ de manière à obtenir une concentration de $1000 \mathrm{mg} . \mathrm{L}^{-1}$ (1000 ppm). A partir de cette solution-mère, des dilutions sont faites, à l'aide de pipettes automatiques Eppendorf type Genex-Alpha.

\section{Analyse de l'huile essentielle}

Un appareil de chromatographie en phase gazeuse de type HP 5890, équipé d'un détecteur, d'un injecteur, d'une colonne apolaire et polaire alternativement, nous a permis de faire l'analyse de l'HE. La flamme 
du détecteur est entretenue par un mélange Hydrogène / Air à des débits de 30 et 300 $\mathrm{mL} / \mathrm{min}$ respectivement. L'analyse au GC/MS est réalisé sur un équipement Agilent 5973 muni d'une colonne apolaire HP1 (MS 60m x $0,25 \mathrm{~mm} \times 0,25 \mu \mathrm{m})$ à une température initiale de $60{ }^{\circ} \mathrm{C}$ en isotherme pendant $10 \mathrm{~min}$, puis à une température finale de $300{ }^{\circ} \mathrm{C}$ pendant 20 min, le gradient étant de $2{ }^{\circ} \mathrm{C} / \mathrm{min}$. Le débit du gaz vecteur (hélium) est de $1 \mathrm{~mL} / \mathrm{min}$. Le détecteur du spectromètre de masse est de type HED/EM (High energy dynode/electron multiplier $0-3000 \mathrm{~V}$ ) avec une énergie de $70 \mathrm{eV}$; les autres paramètres restant identiques.

\section{Evaluation du pouvoir antioxydant \\ Test de réduction du radical DPPH}

Pour le test à la DPPH, les radicaux

$\left(\mathrm{R}^{*}\right)$ sont réduits et protonés comme suit :

$\mathrm{DPPH}^{\bullet}+\mathbf{A H} \rightarrow \mathbf{D P P H}-\mathrm{H}+\mathbf{A}^{\bullet}$ (avec AH : antioxydant)

A cause de sa forte instabilité, le réactif de DPPH est préparé quotidiennement à la concentration 98-100 micromoles par litre. Pour s'assurer de l'exactitude de l'expérimentation, l'absorbance de la solution est lue après chaque préparation à l'aide du spectrophotomètre (DMS 300 UV-visible spectrophotometer), à la longueur d'onde de $517 \mathrm{~nm}$. Une densité optique (DO) lue, comprise entre 0,9-1,0 atteste que le réactif n'est pas encore dégradé. Les titres des solutions testées se situent dans l'intervalle [0$1000 \mathrm{ppm}$ ] alors que la quercétine, la molécule de référence, est utilisée aux concentrations inférieures à $40 \mathrm{ppm}$. Trois essais sont effectués pour chaque titre donné et la moyenne des densités optiques est déterminée. A partir des concentrations des huiles essentielles, des densités optiques lues et des pourcentages d'inhibition calculés, l'intensité colorante $\mathrm{IC}_{50}$ est déterminée. $\mathrm{IC}_{50}$ représente la quantité de produit nécessaire pour faire diminuer la coloration de la solution de réactif (DPPH) de moitié, soit $50 \%$. Pour un échantillon donné, le pouvoir antioxydant est supérieur à celui de la référence si son intensité colorante est inférieure à celle de la quercétine.

Test FRAP

Le pouvoir réducteur des HE est déterminé par cette méthode (Benzie et al., 1999). C'est la capacité totale en antioxydant qui est exprimée par cette méthode. A partir d'une solution mère de $\mathrm{Fe}^{2+}(2000 \mu \mathrm{mol}$ par litre), des dilutions sont faites pour le tracé de la courbe étalon. A $1500 \mu \mathrm{L}$ du réactif dans un tube à essai, on ajoute $150 \mu \mathrm{L}$ d'eau distillée et $50 \mu \mathrm{L}$ de la solution de $\mathrm{Fe}^{2+}$. Le mélange est agité au vortex (marque Heidolph, type REAX 1) et la densité optique est lue après 5 minutes au spectrophotomètre. Une courbe d'étalonnage est ainsi réalisée comme le montre la Figure 1. Pour les échantillons à tester, on mélange dans les mêmes proportions que pour le tracé de la courbe standard, le réactif $(1500 \mu \mathrm{L})$, l'eau distillée $(150 \mu \mathrm{L})$ et la solution du composé à tester $(50 \mu \mathrm{L})$ de titre $1000 \mu$ mol. $^{-1}$. La densité optique est lue après 5 minutes à 593 nm. La capacité «donneur d'électrons »de l'échantillon est mesurée par le changement de couleur lié à la formation du complexe $\left(\mathrm{Fe}^{2+}\right.$ TPTZ) et l'équivalent en $\mathrm{Fe}^{2+}$ est déterminé.

\section{RESULTATS}

Le rendement de l'extraction de l'HE de Vetiveria zizanioides (Poaceae) est de $1,67 \%$ par rapport à la matière sèche des racines. L'huile essentielle de Xylopia aethiopica (Annonaceae) extraite à partir de fruits secs de la plante, de couleur jaune, a un rendement de 3,5\% par rapport à la matière sèche. Aeollanthus pubescens donne une huile essentielle jaune-pâle de rendement $0,20 \%$ à partir des feuilles fraîches et $0,48 \%$ à partir des feuilles sèches. Les composés majoritaires et leurs pourcentages sont répertoriés dans le Tableau 1. Ce sont: Thymol (46,72\%) pour l'HE d'Aeollanthus pubescens, khusimol $(18,68 \%)$ pour l'HE de Vetiveria zizanioides, Linalol $(53,43 \%)$ pour l'HE d'Ocimum basilicum et $\beta$-pinène $(31,92 \%)$ pour l'HE de Xylopia aethiopica. Le Tableau 2 montre que 
tous les extraits d'HE présentent des activités antioxydantes différentes.

Dans notre étude, il y a deux échantillons qui se remarquent par leurs pouvoirs antioxydants indépendamment des tests utilisés. Il s'agit d'Ocimum basilicum et Aeollanthus pubescens (Tableau 2). Pour ces deux HE, les DO varient considérablement $(0,7$ - 0,2) (Figure 2) alors que les densités optiques de certains échantillons ne changent pratiquement pas (de 0,7 à 0,6) (Figure 3) au cours du test à la DPPH. Ocimum basilicum à linalol est l'échantillon le plus antioxydant dans le groupe étudié avec une $\mathrm{IC}_{50}$ de 266 ppm (Figure 2) et un équivalent en $\mathrm{Fe}^{2+}$ de $1318 \mu \mathrm{mol} . \mathrm{L}^{-1}$. Même une durée plus importante d'incubation, n'a aucun effet sensible de changement sur la densité optique de la solution de l'huile essentielle de Xylopia aethiopica. Dans ce cas, la densité optique de la solution de l'échantillon à analyser varie très peu par rapport à celle du blanc constitué par la DPPH en solution dans le méthanol. Les résultats trouvés montrent que dans le groupe, cette HE a un pouvoir antioxydant négligeable. Pour des échantillons avec un pouvoir remarquable, comme Ocimum basilicum, les densités optiques sont fortement dépendantes aussi bien de la concentration que du temps d'incubation et les équivalents en $\mathrm{Fe}^{2+}$ sont élevés. Nous avons utilisé pour nos calculs les moyennes arithmétiques des densités optiques lues pour les trois essais.

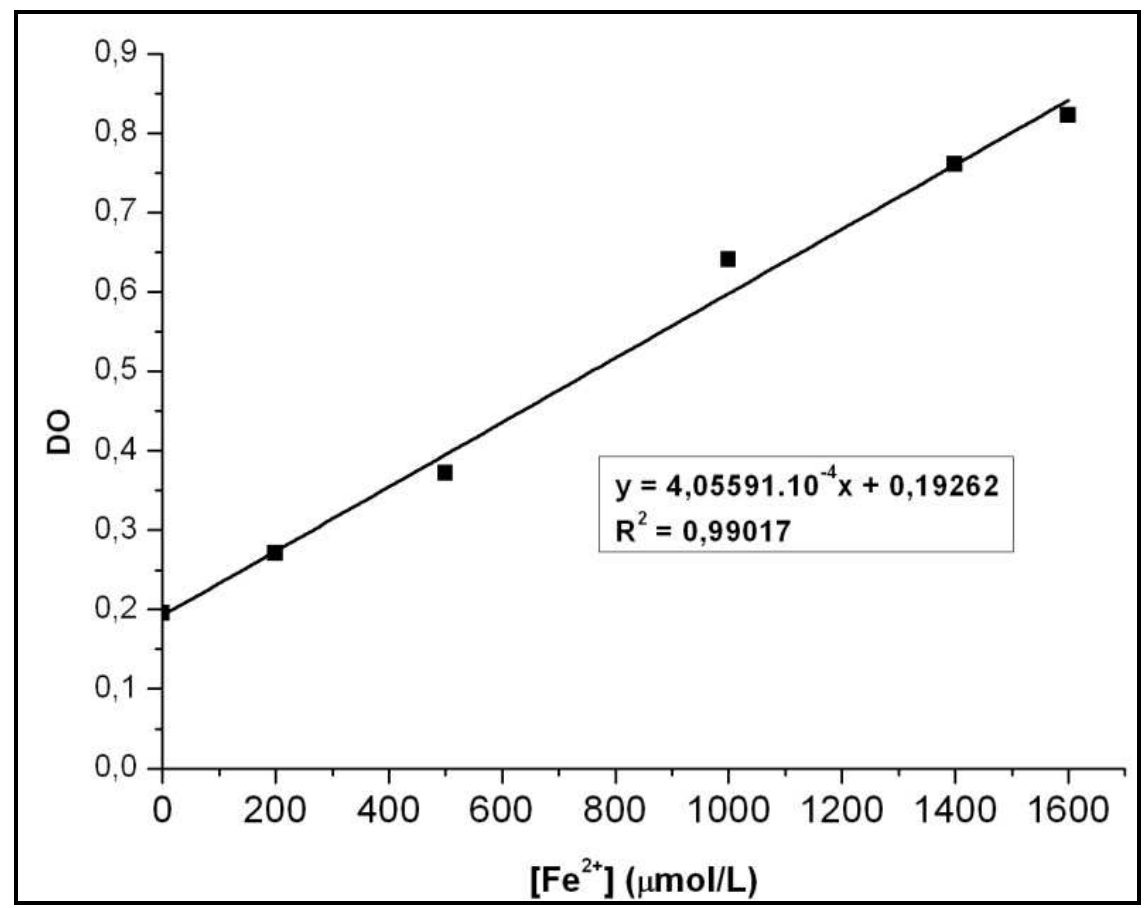

Figure 1 : Variation de la densité optique en fonction de la concentration en $\mathrm{Fe}^{2+}(\mu \mathrm{mol} / \mathrm{l})$. DO sur la figure représente la densité optique lue. « $\mathrm{Y} »$ représente l'équation de la droite et « $\mathrm{R} »$ est le coefficient de corrélation. 


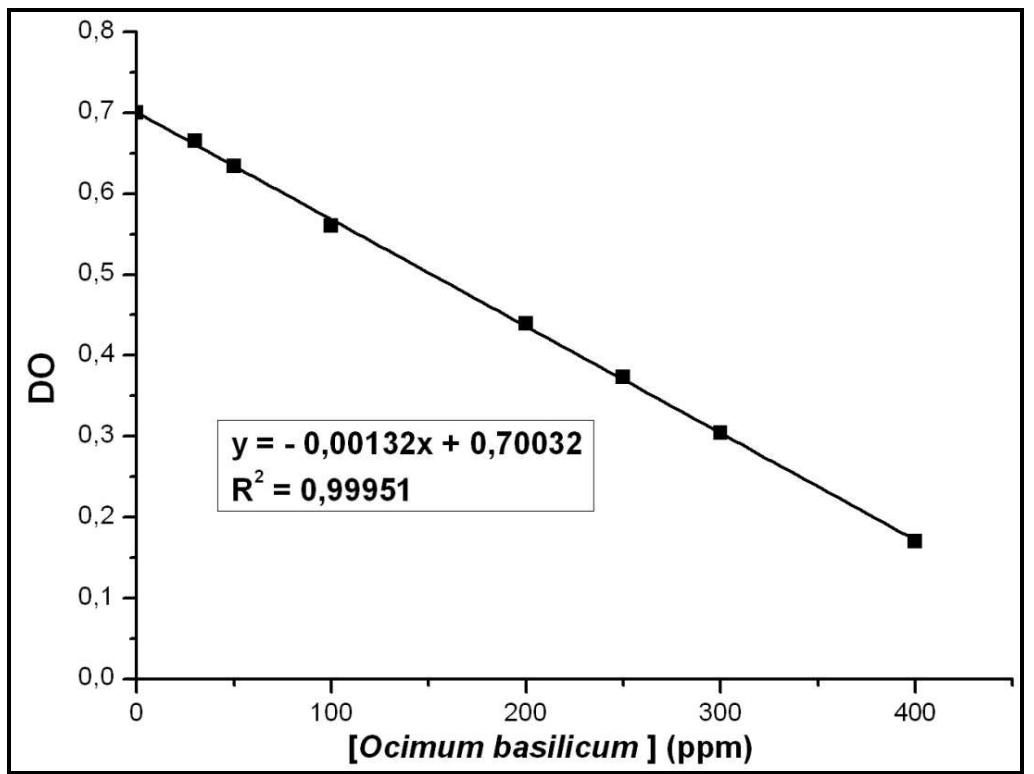

Figure 2: Variation de la densité optique en fonction de la concentration de l'HE d'Ocimum basilicum. DO sur la figure représente la densité optique lue. L'unité partie par million est notée ppm. «Y » représente l'équation de la droite et « $\mathrm{R}$ » est le coefficient de corrélation.

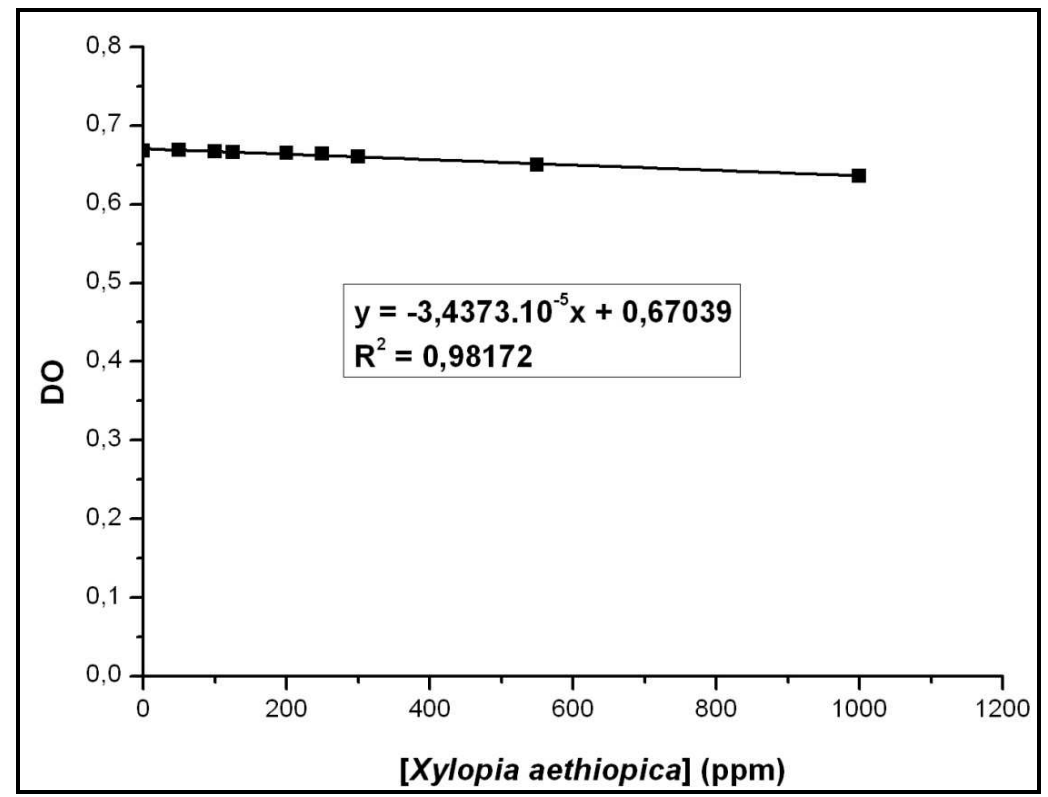

Figure 3 : Variation de la densité optique en fonction de la concentration de l'HE de Xylopia aethiopica. DO sur la figure représente la densité optique lue. L'unité partie par million est notée ppm. «Y» représente l'équation de la droite et « $\mathrm{R}$ » est le coefficient de corrélation. 
Tableau 1: Composés majoritaires et leurs pourcentages dans les échantillons testés.

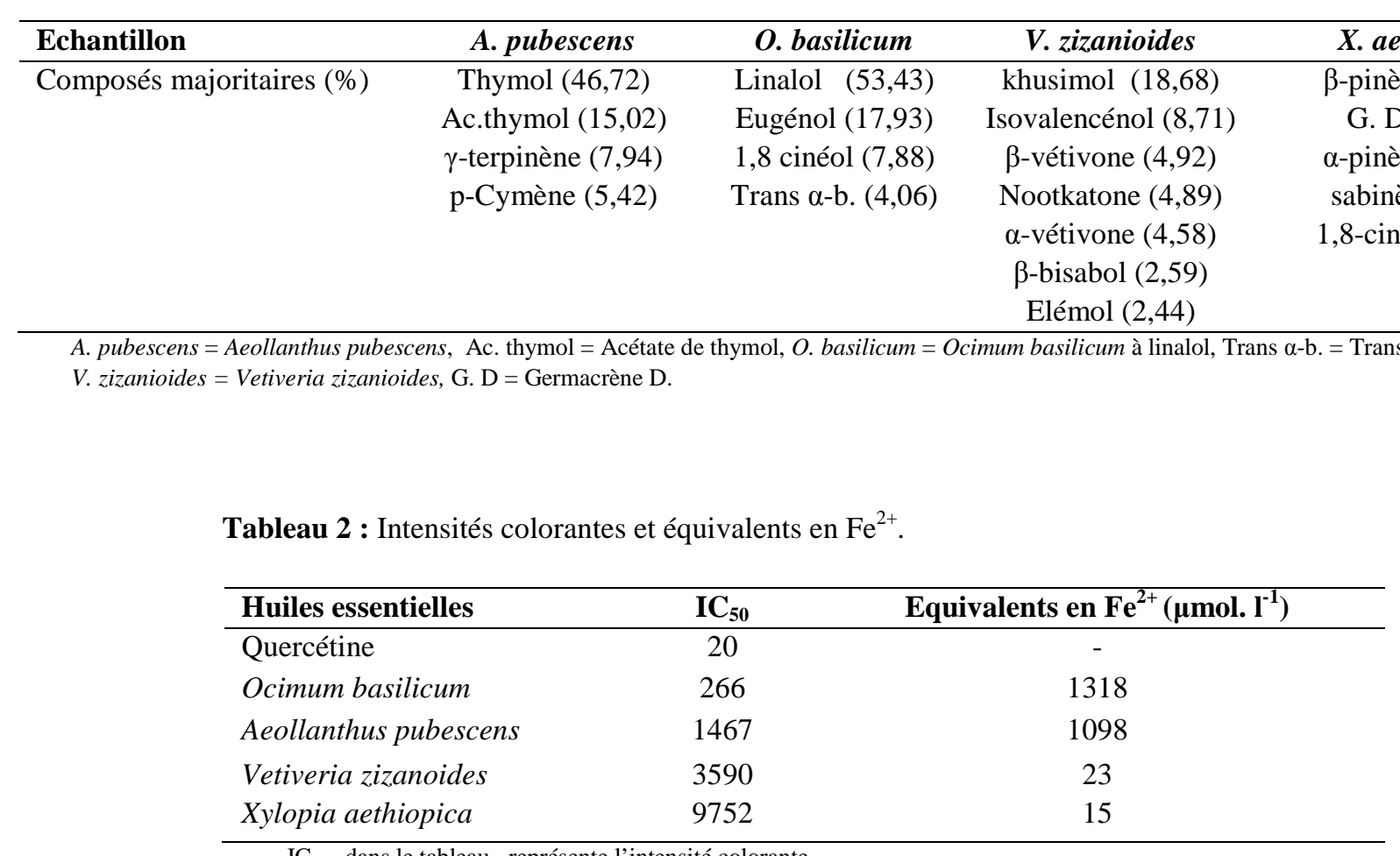




\section{DISCUSSION}

Notre étude a porté sur les échantillons répertoriés dans le Tableau 1 et les intensités colorantes avec les équivalents en $\mathrm{Fe}^{2+}$ des échantillons testés figurent dans le Tableau 2. Nous notons que l'efficacité de réduction du fer suivant la méthode FRAP est inversement proportionnelle à la valeur $\mathrm{IC}_{50}$ déterminée par le test à la DPPH. Le classement est le suivant : Ocimum basilicum > Aeollanthus pubescens $>$ Xylopia aethiopica $>$ Vetiveria zizanioides. $\mathrm{La}$ concentration $\mathrm{IC}_{50}$ la plus faible est enregistrée pour Ocimum basilicum et vaut $266 \mathrm{ppm}$ et la plus élevée $3590 \mathrm{ppm}$ pour Vetiveria zizanioides. Ces résultats permettent de conclure que l'HE d'Ocimum basilicum présente une meilleure activité antioxydante, par rapport aux autres HE testées, suivie de l'HE d'Aeollanthus pubescens. L'HE de Xylopia aethiopica, avec un pouvoir antioxydant faible, est prioritairement composée d'hydrocarbures non aromatiques ( $\beta$-pinène $=27,68 \%, \alpha$ pinène $=9,54 \%)$. Aeollanthus pubescens a majoritairement dans sa composition des composés aromatiques (Thymol (46,72\%), Acétate de thymol $(15,02 \%)$ ) de même que l'HE d'Ocimum basilicum (Linalol $(53,43 \%)$, Eugénol $(17,93 \%))$. La présence des composés antioxydants dans les extraits d'HE diminue l'absorbance en fonction du temps, de 0,7 à 0,2 pour l'HE d'Ocimum basilicum. Cette absorbance ne varie presque pas pour les solutions d'HE qui ont un faible pouvoir antioxydant, de l'ordre de 0,65 pour l'HE de Xylopia aethiopica. La courbe illustrée dans la Figure 2 nous montre que l'HE d'Ocimum basilicum est capable de neutraliser le radical DPPH en fonction de la concentration. L'activité d'un antioxydant est directement liée à la concentration. Il serait intéressant, dans nos études futures, de trouver les concentrations qui donneraient un effet bénéfique pour nos $\mathrm{HE}$ avec une capacité antioxydante remarquable. Il est courant de trouver dans la littérature scientifique que les composés phénoliques possèdent une forte capacité antioxydante. Toutefois, Il est très accepté que ce n'est pas nécessairement la forte teneur en polyphénols qui confère une activité antioxydante remarquable (Moure et al., 2001). C'est pour cela que certains auteurs calculent le paramètre $\mathrm{EC}_{50}$ qui est une mesure quantitative directe pour l'activité antioxydante. Il est défini comme la concentration de l'extrait brut des composés phénoliques nécessaire pour réduire 50\% de la concentration initiale du DPPH (Benhammou, 2012). Dans notre étude nous pensons plutôt à une action synergique du thymol $(46,72 \%)$ et de l'acétate de thymol $(15,02 \%)$ dans l'HE d'Aeollanthus pubescens d'une part et du linalol $(53,43 \%)$, de l'eugénol $(17,93 \%)$ et du 1,8 cinéol $(7,88 \%)$ dans l'HE d'Ocimum basilicum d'autre part. On note dans l'HE de Vetiveria zizanioides, l'effet contraire, un antagonisme des composés majoritaires (khusimol (18,68\%) et Isovalencénol $(8,71 \%)$ ) qui ne permet pas une bonne capacité antioxydante. La forte teneur en hydrocarbures, $(\beta$-pinène $=27,68 \%, \alpha$-pinène $=9,54 \%)$ dans l'HE de Xylopia aethiopica, pourrait expliquer son pouvoir antioxydant insignifiant. Les données du Tableau 2 montrent que tous les échantillons d'HE étudiés sont moins antioxydants que la molécule de référence.

\section{Conclusion}

Miliauskas et al. (2004) ont montré que le classement, dans un groupe donné du pouvoir antioxydant, dépend de la méthode utilisée. Toutefois, nos résultats ne montrent pas des comportements distincts par rapport à la méthode DPPH et au test FRAP pour le même échantillon. Les résultats de notre étude montrent que le classement n'est pas différent selon la méthode utilisée. On remarque que toutes les HE étudiées sont moins antioxydants que la quercétine (Tableau 2) qui a une $\mathrm{IC}_{50}$, dans nos conditions d'expérience, voisine de $20 \mathrm{ppm}$ indépendamment des moyens de calcul utilisés (pourcentage d'inhibition ou densité optique). L'équivalent en $\mathrm{Fe}^{2+}$ de la quercétine est très élevé, ce qui ne peut être déterminé à partir de l'intervalle 
considéré de la courbe standard utilisée. Ces résultats préliminaires nous révèlent deux $\mathrm{HE}$, (Ocimum basilicum à linalol et Aeollanthus pubescens) testées, possédant une activité antioxydante remarquable in vitro. D'autres études sont nécessaires pour évaluer le potentiel in vivo de ces huiles sur des animaux (test de toxicité par exemple) avant d'envisager la possibilité de leur utilisation dans l'industrie agroalimentaire et en médecine.

\section{REFERENCES}

Benhammou N. 2011. Activité antioxydante des extraits des composés phénoliques de dix plantes médicinales de l'Ouest et du Sud-Ouest Algérien. Thèse de doctorat, Université Aboubakr Belkaïd-Tlemcen, Algérie.

Benzie IFF, Strain JJ. 1999. Ferric reducing antioxidant power assay: direct measure of total activity of biological fluids and modified version for simultaneous measurement of total antioxidant power and ascorbic acid concentration. Methods Enzymol., 299: 15-27.

Corinne G. 2013. Additifs Alimentaires, le Guide Indispensable pour ne plus vous Empoisonner. Ed. Chariot d'Or. France.
Djeridane A, Yousfi Y, Brunel JM, Stocker P. 2010. Isolation and characterization of a new steroid derivative as a powerful antioxidant from Cleome arabica in screening the in vitro antioxidant capacity of 18 Algerian medicinal plants. Food Chem Toxicol., 48: 2599-2606.

Hélène BdV. 2008. Les Additifs Alimentaires, ce que Cachent les Etiquettes. Ed. Trédaniel Poche. Paris.

Kokouvi D. 2009. Extraction de l'huile essentielle de Vetiveria zizanioides: performances de la méthode de piégeage à l'hexane et caractérisation de l'huile. Mémoire de diplôme d'ingénieur des travaux en gestion de l'eau et de l'environnement, Université de Lomé, Togo.

Miliauskas G, Venskutonis PR, van Beek TA. 2004. Screening of radical scavenging activity of some medicinal and aromatic plant extracts. Food Chem., 85: 231-237.

Moure A, Cruz JM, Franco D, Dominguez JM, Sineiro J, Dominguez H, Núñez MJ, Parajo JC. 2001. Natural antioxidants from residual sources. Food Chem., 72: 145-171. 